\title{
Primary immunodeficiency diseases in two neighboring pediatric centers: registry data bring out a wide spectrum of diseases with complex clinical presentations
}

\author{
ANGELA PIRRONE ${ }^{\prime}$, GAŠPER MARKELJ ${ }^{2}$, ELISA PISCIANZ ${ }^{3}$, ANJA KOREN JEVERICA ${ }^{2}$, \\ ERICA VALENCIC ${ }^{3}$, MARUŠA DEBELJAK ${ }^{2}$, ALBERTO TOMMASINI ${ }^{3}$, TADEJ AVCIN ${ }^{2}$
}

${ }^{1}$ Department of Pediatrics, University of Trieste, Trieste, Italy

${ }^{2}$ University Children's Hospital, University Medical Center, Ljubljana, Slovenia

${ }^{3}$ Institute for Maternal and Child Health I.R.C.C.S. Burlo Garofolo, Trieste, Italy

\begin{abstract}
In the last decades, the spectrum of primary immunodeficiency diseases (PIDs) has greatly widened, including disorders that can variably impair different immune functions. Although several case series have been published for each disorders, no data is available on how these changes have reflected in the clinical practice of pediatric departments.

Aim of the study: Based on the analysis of registry data, we evaluated the distribution of diagnoses among different PID categories, the clinical features and diagnostic investigations at disease onset in two pediatric departments, namely in Slovenia and in Italy.

Results: 136 patients have been diagnosed at the two centers, with a widespread distribution into different disease categories. Considering the 109 patients who were still alive at the last follow-up, prevalence of pediatric-onset-PID in our area was roughly estimated to be around 31 per million inhabitants. Diagnosis was genetically confirmed in 79 cases (58.1\%), with 29 different genes found mutated. The most common presenting symptoms were: recurrent infections $(52.2 \%)$, inflammatory manifestations (36.7\%), specific syndromic features (30.8\%), unusual infections $(1.6 \%)$ and failure to thrive/growth retardation (22.8\%). Treatments at follow-up include antimicrobials (20), hematopoietic stem cell transplantation (17), immunoglobulin replacement therapy (16), and immunosuppressants (9).

Conclusions: A huge number of different PIDs are encountered in pediatric departments, often presenting with complex clinical pictures. Our results suggest that the identification of PID may be improved by a multidisciplinary approach, attaching importance not only to infections but also to other symptoms arising from a defective immune function.
\end{abstract}

Key words: primary immunodeficiency, warning signs, infections, autoimmunity, inflammation, immune dysregulation.

(Centr Eur J Immunol 2012; 37 (4): 365-370)

\section{Introduction}

In the last few decades, the spectrum of primary immunodeficiencies (PIDs) has greatly widened, including more than 150 different definite entities [1], and this is slowly being reflected in the distribution of PID in international registries [2]. Besides classical PIDs, new disorders are being increasingly diagnosed, which are associated with complex defects of immune functions [3]. Nowadays, it is a well accepted concept that the clinical spectrum of PID includes inflammatory, autoimmune and lymphoproliferation symptoms as well as recurrent or severe infections. Indeed, even disorders classically characterized by infections may present at first with non-infectious symptoms [4].

Correspondence: Alberto Tommasini, MD, PhD, Laboratory of Immunopathology, Institute for Maternal and Child Health, IRCCS Burlo Garofolo, via dell'Istria 65/1, 34137 Trieste, Italy 
As a consequence, warning signs developed many years ago to improve the recognition of PIDs - such as the 10 signs promoted by the Jeffrey Modell Foundation - may be insufficient today, in particular in pediatric practice [5]. The European Society for Immunodeficiencies (ESID) proposed a different set of warning criteria that should include inflammatory, autoimmune and lymphoproliferative features, as well as other long-established criteria, to allow a better identification of PIDs by non immunologists [6]. However, the consequences of these developments in the daily clinical practice of pediatric departments have not been analyzed so far. On the other hand, according to recent data, the specific training of physicians on PID seems to have a major role in improving recognition and diagnosis of these disorders $[7,8]$.

Herein, we are discussing the experience with PIDs at two neighboring pediatric centers, in Slovenia and in NorthEast Italy, serving together an area of about 3.5 million inhabitants. Clinical records were analyzed to estimate the prevalence of each disorder, the clinical features at disease onset, the laboratory investigations that led to diagnosis and the treatment at last follow-up.

\section{Material and methods}

\section{Study design}

Analysis of all patients included in the two registries of PID from two neighboring pediatric centers. Trieste registry was created in June 2007 and included all patients diagnosed with a PID in the Friuli Venezia Giulia area, in NorthEast Italy. The Slovenian National registry was established in November 2007 and includes all patients diagnosed with a PID in Slovenia. At the time of the establishment of the two registries, medical records of all patients previously diagnosed with a PID were retrospectively reviewed by one of the authors. Since the registries were established, the data of all new patients were prospectively collected until January 2011 .

To be eligible for enrollment into this study, patients had to be diagnosed as having PID before the age of 18. We selected only patients coming from Slovenia and Friuli Venezia Giulia, to allow a rough estimate of the real prevalence of each disorder. A further series of patients referred for diagnosis from other areas has been analyzed separately. Our patients were classified into the following eight categories, according to the International Union of Immunological Societies classification [1]: predominantly antibody disorders; predominantly T-cell deficiencies; phagocytic disorders; complement deficiencies; other well defined PIDs (also indicated as "syndromic PIDs"); autoimmune \& immune dysregulation syndromes; autoinflammatory syndromes; unclassified immunodeficiency diseases.

The diagnosis of PID was based on clinical features and on laboratory investigations, including analyses of lymphocyte subsets by cytometry, studies of lymphocyte acti- vation, proliferation and apoptosis, studies of the reactive burst of neutrophils, analyses of complement levels of immunoglobulins and specific antibodies, and sequence analyses of candidate genes.

In some cases, blood samples of patients were sent to the different laboratories within the ESID network for further immunological investigation or specific genetic analysis.

Our analysis particularly focused on serious immunodeficiency diseases requiring complex therapeutic approaches. Patients presenting transient infancy's hypogammaglobulinemia, immunoglobulin G (IgG) subclasses deficiency and/or selective IgA deficiency were not included in our study because these disorders are too common and in most cases characterized by mild and non-specific complaints.

For each patient, clinical data at the time of diagnosis and during the follow up were recorded. Manifestations considered suspicious of PID were analyzed in each patient and compared with the eight warning criteria suggested by the Clinical Working Party of the European Society for Immunodeficiencies [6]. The familial history was considered a criterion of PID too, even though it is not specifically included in the ESID criteria set.

All patients were directly evaluated by, at least, one of the authors. All symptoms that occurred before diagnosis were recorded as "presenting symptoms". For analytical convenience, the following groups of symptoms were considered: 1) autoimmune chronic inflammatory disease and/or lymphoproliferation, which included the following: autoimmune disorders; non-infectious enlargement of lymphnodes/spleen/liver; chronic inflammatory disorders of the intestine, joint, vessels; diffuse dermatitis; alopecia; autoimmune cytopenias; 2 ) infection-related criteria included: recurrent ear, nose, throat and airway infections (mainly due to extracellular bacteria, enterovirus or Giardia); recurrent pyogenic infections; unusual infections (mainly due to intracellular bacteria or fungi) or unusually severe course of infections; recurrent infections with the same type of pathogen; 3) failure to thrive from early infancy or growth retardation not explained otherwise; 4) characteristic combinations of clinical features in eponymous syndromes (see [9]). The genetic examinations performed in the diagnostic path were also recorded.

The study was approved by the independent Ethics Committees of the IRCCS Burlo Garofolo, Trieste and the Slovenian Ministry of Health.

\section{Results}

In the period from January 1975 to December 2011, a total of 176 patients (104 males) were diagnosed with a PID at the two Institutes; of these 136 (85 males) come from the reference area. At the time of the study, 109 patients were still alive.

The incidence of diagnoses progressively increased until the last decade, and as much as $45.6 \%$ of the patients have 
been diagnosed in the last 4 years, after the two PID registries were established. A summary of the patients' characteristics is given in Table 1 .

Considering a population of about 3.5 million inhabitants in the local area, and considering that the two Institutes are the only referral centers in this area, a rough prevalence of pediatric onset PID is estimated to be around 31 per million.

Overall, 36 different disorders have been diagnosed, distributed in all eight categories (predominantly antibody defects $9.6 \%$, combined defects $13.2 \%$, phagocyte deficiencies $18.4 \%$, other well defined (or syndromic) PIDs $23.5 \%$, dysregulatory disorders $19.9 \%$, complement defects $5.9 \%$ and autoinflammatory diseases $9.6 \%$ ).

A genetic diagnosis was obtained in 79 cases (58.1\%), with mutations identified in 29 different genes. This complexity of the case series is resumed in Table 2.

Chronic granulomatous disease (15 cases, $11.0 \%$ in 14 unrelated families, with X-linked inheritance in 11 cases) was the most common single disorder. Autoimmune polyendocrinopathy candidiasis ectodermal dystrophy APECED (10 cases in 9 unrelated families) and familial hemophagocytic lymphohistiocytosis - fHLH (8 cases in 7 unrelated families) were also common disorders in this series and they were never attributable to consanguinity.

Average delay in diagnosis from the first symptoms was 17 months. Children with 22q11 deletion syndrome, severe combined immunodeficiency (SCID), f-HLH and Wiskott Aldrich syndrome showed a shorter average delay in diagnosis. Patients with X-linked agammaglobulinemia, milder combined deficiencies, APECED and X-linked lymphoproliferative disease showed the longest average delay in diagnosis.

Unusual and/or recurrent infections contributed to the clinical picture before diagnosis in 96 patients (overall 70.6\%; recurrent infections in $52.2 \%$ and unusual infections in $30.9 \%$ of cases) (Fig. 1). Inflammatory, autoimmune or lymphoproliferation symptoms were present in 74 cases (overall $54.4 \%$ : chronic inflammation in $35.3 \%$, autoimmunity in $14.7 \%$ and lymphoproliferative disorders in $9.5 \%$ ). Specific syndromic features were present in $29.4 \%$ of patients (dysmorphic features in 10 patients; ectodermal anomalies in 8 ; micro-thrombocytopenia in 6; neurodevelopmental defects in 7; other syndromic features in 9; angioedema in 1). Failure to thrive or growth retardation were reported in 30 patients $(22.1 \%)$ and neoplasm in 7 (5.1\%).

A familial history of primary immunodeficiency was present in 9 patients $(6.6 \%$; 7 patients with an autosomal recessive pattern and 2 with $\mathrm{X}$-linked transmission). In no case consanguinity was reported.

Inflammatory bowel disease (IBD) was the most common inflammatory complaint (12.5\% of patients), followed by unexplained long lasting or recurrent fevers $(10.3 \%)$, rheumatic diseases $(5.9 \%)$ and dermatitis $(5.1 \%)$. While IBD and dermatitis were more common in children younger than 1 year of age, unexplained fever was registered at all ages but rheumatic disorders only in older children.
At follow-up, 20 patients were treated with antimicrobials, 17 had a stable engraftment of hematopoietic stem cell from donors, 16 were given immunoglobulin replacement therapy, and 9 patients were treated with immunosuppressants and/or biological agents.

\section{Discussion}

We herein described how new knowledge on PIDs impacted on the clinical experience of two medium-sized pediatric centers.

We roughly calculated a prevalence of PID of around 31 cases per million inhabitants. This is probably underestimated, considering that a percentage of PID can still go undiagnosed or that some cases can be referred to other centers for any reason. However, considering that we only collected PID reported in pediatric age, this percentage is consistent with the expectations [10-15].

In our series, primary immune deficiencies are widely distributed in all eight disease categories according to the most commonly used international classification [1]. In particular, the disease categories of "dysregulatory disorders" and "auto-inflammatory disorders" - which were poorly represented or completely absent in previous series - included cumulatively $29.5 \%$ of patients, while "predominantly antibody deficiencies" - which constitute the largest group in most published series - includes as little as $9.6 \%$ of patients. This is probably due to several causes.

Firstly, common variable immunodeficiency is in absolute terms the most common immunodeficiency and is responsible for the largest part of antibody defects. This disorder, however, is typically present in adults and, as a consequence, is scarcely represented in our series.

Secondly, the epidemiology of PIDs is changing, thanks to an increased awareness of the problem and to the availability of new immunological and genetic assays. As a matter of fact, recent registries present a wider distribution of diagnoses in all categories [2].

Thirdly, some disorders classified as PID are primarily cared for by different specialists (rheumatologists, hematologists, endocrinologists, dermatologist) and are more likely to be under-represented in registries held by immunologists. This was not the case in our study, as the practice of multiple disciplines in the same pediatric department easily allowed to collect all cases together. For example, APECED seemed to be more common in our series compared to other PID registries. However, the prevalence of this disorder is not so high when data are compared to registries held by endocrinologists [16].

Lastly, some disorders, such as auto-inflammatory diseases, are probably still under-diagnosed in many centers. Indeed, auto-inflammatory and dysregulatory disorders represent the most common PID diagnoses among patients referred to our clinics from other areas (overall, $70 \%$ of the patients referred from other areas, data not shown). 
One of the aims of our study was to analyze the prevalence of different symptoms at disease presentation in each PID category. This was possible thanks to the fact that all registered patients had been cared for by at least one of the authors and that near half of the cases had been collected prospectively. For a better analysis, we decided to refer each symptom to one of the six ESID warning criteria for PID [6]. As expected, recurrent or unusual infections represented the most common presenting symptom in patients affected by PIDs, being reported in $70.4 \%$ of patients. The detection of

Table 1.

\begin{tabular}{|c|c|c|c|c|c|c|c|c|}
\hline & Patients & $(\%)$ & Male & $\begin{array}{c}\text { Age at } \\
\text { presentation; } \\
\text { median } \\
\text { and range } \\
\text { (months) }\end{array}$ & $\begin{array}{c}\text { Delay to } \\
\text {; diagnosis; } \\
\text { median } \\
\text { and range } \\
\text { (months) }\end{array}$ & $\begin{array}{l}\text { Positive } \\
\text { genetic } \\
\text { results }\end{array}$ & $\begin{array}{l}\text { Alive in } \\
\text { follow-up }\end{array}$ & $\begin{array}{c}\text { Diagnosis \% } \\
\text { in the last } \\
4 \text { years }\end{array}$ \\
\hline Prevalent antibody deficiencies & 13 & 9.6 & 8 & $24(1-144)$ & $48(4-71)$ & 6 & 12 & $38.5 \%$ \\
\hline common variable immune deficiency & 7 & 5.1 & 2 & $120(6-144)$ & $12(7-60)$ & 0 & 6 & \\
\hline X-linked agammaglobulinemia & 6 & 4.4 & 6 & $16(1-84)$ & $54(4-71)$ & 6 & 6 & \\
\hline Combined immunodeficiencies & 18 & 13.2 & 11 & $2(0-48)$ & $12(0-66)$ & 7 & 14 & $55.6 \%$ \\
\hline combined immune deficiencies & 12 & 8.8 & 6 & $2(0-48)$ & $53(2-66)$ & 2 & 9 & \\
\hline severe combined immune deficiencies & 6 & 4.4 & 5 & $2(1-16)$ & $5(0-20)$ & 5 & 5 & \\
\hline Phagocyte deficiencies & 25 & 18.4 & 21 & $4(0-60)$ & $15(0-120)$ & 15 & 20 & $36.0 \%$ \\
\hline chronic granulomatous disease & 15 & 11.0 & 15 & $5(1-60)$ & $23(0-120)$ & 11 & 10 & \\
\hline Shwachman-Diamond syndrome & 5 & 3.7 & 3 & $3(0-36)$ & $14(3-120)$ & 4 & 5 & \\
\hline others & 5 & 3.7 & 3 & $6(3-20)$ & $4(0-128)$ & 1 & 5 & \\
\hline Complement deficiencies & 8 & 5.9 & 3 & $42(8-186)$ & $6(0-10)$ & 5 & 8 & $50.0 \%$ \\
\hline Other well defined ID & 32 & 23.5 & 19 & $6(1-135)$ & $9(0-179)$ & 16 & 24 & $46.9 \%$ \\
\hline Wiskott-Aldrich syndrome & 6 & 4.4 & 6 & $2(1-5)$ & $6(0-49)$ & 6 & 4 & \\
\hline ataxia telangectasia & 5 & 3.7 & 3 & $21(2-30)$ & $28(6-66)$ & 0 & 3 & \\
\hline 22q11-deletion syndrome & 5 & 3.7 & 0 & $0(0-7)$ & $1(0-1)$ & 5 & 5 & \\
\hline hyper IgE syndrome & 3 & 2.2 & 2 & $7(0-10)$ & $17(0-32)$ & 1 & 3 & \\
\hline Njimegen breakage syndrome & 3 & 2.2 & 2 & $36(24-135)$ & $36(2-78)$ & 1 & 1 & \\
\hline others & 10 & 7.4 & 6 & $3(1-11)$ & $24(0-179)$ & 4 & 6 & \\
\hline Immune dysregulation syndromes & 27 & 19.9 & 19 & $18(0-132)$ & $39(0-120)$ & 18 & 19 & $33.3 \%$ \\
\hline APECED & 10 & 7.4 & 5 & $42(36-90)$ & $102(48-120)$ & 9 & 10 & \\
\hline $\begin{array}{l}\text { familial hemophagocitic lympho } \\
\text { histiocytosis }\end{array}$ & 8 & 5.9 & 6 & $3(0-6)$ & $4(1-13)$ & 5 & 3 & \\
\hline $\begin{array}{l}\text { autoimmune lymphopriliferative } \\
\text { syndrome }\end{array}$ & 3 & 2.2 & 3 & $63(9-132)$ & $39(0-60)$ & 1 & 3 & \\
\hline $\mathrm{X}$-linked lymphoproliferative disease & 3 & 2.2 & 3 & $32(0-74)$ & $(42-84)$ & 2 & 1 & \\
\hline others & 3 & 2.2 & $23(1-7)$ & $24(5-84)$ & 1 & 1 & & \\
\hline Autoinflammatory disorders & 13 & 9.6 & 4 & $9(1-132)$ & $15(8-105)$ & 11 & 12 & $76.9 \%$ \\
\hline mevalonate kinase deficiency & 7 & 5.1 & 2 & $6(1-30)$ & $30(8-105)$ & 5 & 5 & \\
\hline familial mediterranean fever & 5 & 3.7 & 1 & $30(3-132)$ & $12(12-121)$ & 5 & 4 & \\
\hline TRAPS & 1 & 0.7 & 1 & 72 & 12 & 1 & 3 & \\
\hline Total & 136 & 100.0 & 84 & $7(1-144)$ & $17(0-179)$ & 78 & 109 & $45.6 \%$ \\
\hline
\end{tabular}


Table 2. Complexity of the PID series

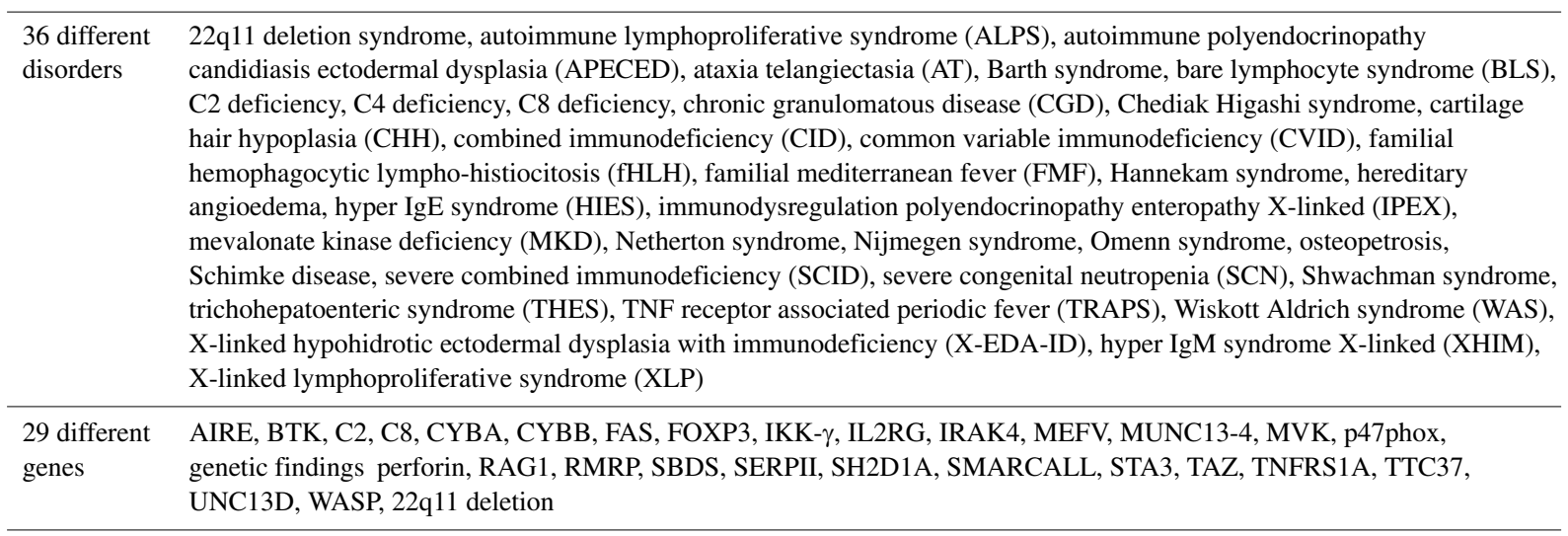

opportunistic pathogens such as Pneumocystis jiroveci, Aspergillus or Serratia was always considered highly suggestive of a PID (a combined immunodeficiency or a phagocyte deficiency), as already well known in literature [17-20].

Unexpectedly, inflammatory, autoimmune and lymphoproliferation symptoms were very common as well (54.4\% of cases), not only in patients with dysregulatory or auto-inflammatory disorders, but through all the categories. The early onset of multiple autoimmune disorders, the presence of inflammatory symptoms poorly responsive to therapies and an unexplained lymphoproliferation were always considered highly suspicious of a PID.

The risk of developing autoimmune or chronic inflammatory diseases and/or lymphoproliferation in PIDs has been widely described [21-25]. However, few studies have evaluated the frequency of these symptoms at disease presentation. Furthermore, it has been increasingly reported that some PIDs, including Wiskott Aldrich disease, severe combined immunodeficiencies and chronic granulomatous disease can present first with inflammation rather than with infections [4, 24, 26-28]. We herein showed that this could be even more common than we thought. Taking into account these results, we would stress the importance of considering symptoms arousing from a dysregulated immunity if a PID is suspected. A practical example of common dysregulatory features associated with PID is given in a recent review [5].

Failure to thrive and poor growth are also important signs supporting the suspect of a PID, as they are present in $22.1 \%$ of patients. Indeed, in one patient affected by a SCID, failure to thrive was the sole symptom before diagnosis.

Presentation symptoms of PID were evaluated in a recent retrospective series in the UK, in order to assess the effectiveness of the 10 warning signs of PID proposed by the Jeffrey Modell Foundation (JMF). The authors found that among the JMF warning signs, "positive family history of PID", "intravenous antibiotic for sepsis" and "failure to thrive" predict at least $89 \%$ of children with T-lymphocyte, complement and neutrophil PID [29]. On the other hand, the same group discussed in a following review that this set of warning signs is not suitable to identify a huge number of PID associated to dysregulatory symptoms and thus a new paradigm should be proposed for the $21^{\text {st }}$ century [5]. Addressing this issue could significantly shorten the diagnostic delay in many patients with PID. In fact, for the "older" PID, such as SCID and well defined syndromic defects - which are easily recognized thanks to typical clinical features - we observed a shorter delay from disease presentation to diagnosis. Furthermore, only one patient with a classical immunodeficiency syndrome was referred to us from other areas, suggesting that knowledge of these disorders is widespread in pediatric departments (data not shown).

However, diagnostic delay is still too long for "new immunodeficiencies", suggesting an insufficient awareness

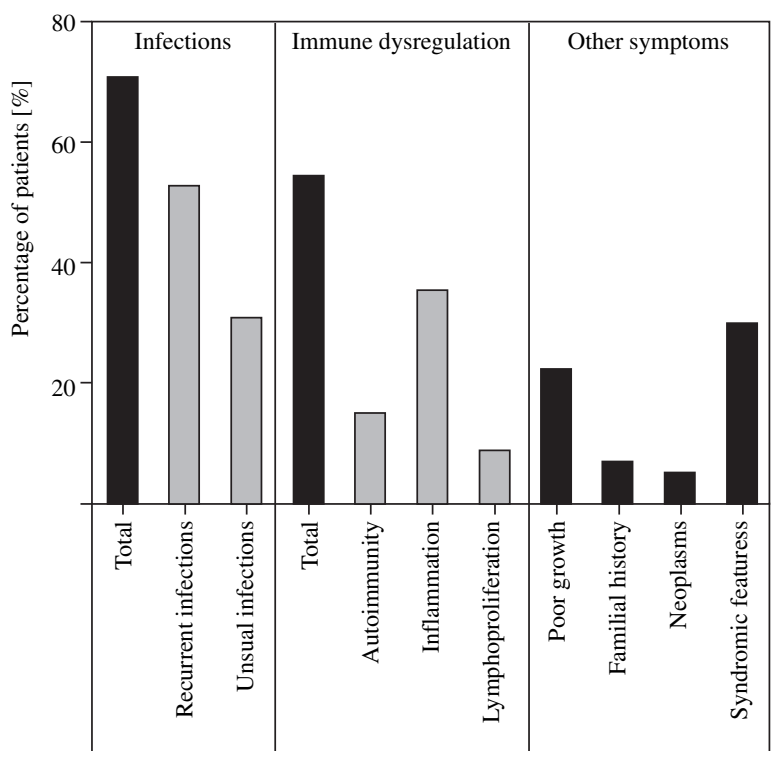

The work was founded by the Institute for Maternal and Child Health, IRCCS Burlo Garofolo, grant \# 03/09 and the grants from the Slovenian Research Agency (L3-4150 and P3-0343).

Fig. 1. Symptoms related to infection, immune dysregulation and other symptoms at presentation 
of these disorders among pediatricians and/or a lack of simple diagnostic assays.

In our series, we noticed that in recent decades the diagnostic delay for many diseases has shortened, probably due to an increased awareness of the problem and to the availability of new immunological and genetic examinations (data not shown).

Another point that clearly emerges from our experience is the growing complexity of PID, with several specific disorders and genes involved. Actually, the application of warning signs may not be sufficient to cope with these changes, unless it is associated to a specific training of the specialist in PID.

In our experience, the best strategy to optimize diagnosis in this field is based on sharing clinical experience (e.g. with case studies) and networking among pediatric departments involved in the field of PID. The analysis of our registries confirms a tendency to an increasing complexity of PIDs in the pediatric population. Careful consideration of immune dysregulation/inflammation symptoms, failure to thrive and familial history, as well as infectious symptoms, is particularly important to avoid delays in diagnosis. We finally suggest that establishment of a formal PID registry can be a precious tool to increase awareness and recognition of these rare disorders.

\section{The authors declare no conflict of interest.}

\section{References}

1. Notarangelo LD, Fischer A, Geha RS, et al. (2009): Primary immunodeficiencies: 2009 update. J Allergy Clin Immunol 124: 1161-1178.

2. CEREDIH (2010): The French national registry of primary immunodeficiency diseases. Clin Immunol 135: 264-272.

3. McGonagle D, Aziz A, Dickie LJ, McDermott MF (2009): An integrated classification of pediatric inflammatory diseases, based on the concepts of autoinflammation and the immunological disease continuum. Pediatr Res 65: 38R-45R.

4. Freudenberg F, Wintergerst U, Roesen-Wolff A, et al. (2010): Therapeutic strategy in $\mathrm{p} 47$-phox deficient chronic granulomatous disease presenting as inflammatory bowel disease. J Allergy Clin Immunol 125: 943-946.

5. Arkwright PD, Gennery AR (2011): Ten warning signs of primary immunodeficiency: a new paradigm is needed for the 21st century. Ann N Y Acad Sci 1238: 7-14.

6. de Vries E (2006): Patient-centred screening for primary immunodeficiency: a multi-stage diagnostic protocol designed for non-immunologists. Clin Exp Immunol 145: 204-214.

7. Maródi L, Casanova JL (2009): Primary immunodeficiency diseases: the J Project. Lancet 373: 2179-2181.

8. Maródi L, Casanova JL (2009): Novel primary immunodeficiencies relevant to internal medicine: novel phenotypes. J Intern Med 266: 502-506.

9. Kersseboom R, Brooks A, Weemaes C (2011): Educational paper: syndromic forms of primary immunodeficiency. Eur J Pediatr 170: 295-308.
10. Leiva LE, Zelazco M, Oleastro M, et al. (2007): Primary immunodeficiency diseases in Latin America: the second report of the LAGID registry. J Clin Immunol 27: 101-108.

11. Knerr V, Grimbacher B (2007): Primary immunodeficiency registries. Curr Opin Allergy Clin Immunol 7: 475-480.

12. Kirkpatrick P, Riminton S (2007): Primary immunodeficiency diseases in Australia and New Zealand. J Clin Immunol 27: 517-524.

13. Gathmann B, Grimbacher B, Beauté J, et al. (2009): The European internet-based patient and research database for primary immunodeficiencies: results 2006-2008. Clin Exp Immunol 157 Suppl 1: 3-11.

14. Abuzakouk M, Feighery C (2005): Primary immunodeficiency disorders in the Republic of Ireland: first report of the national registry in children and adults. J Clin Immunol 25: 73-77.

15. Stray-Pedersen A, Abrahamsen TG, Frøland SS (2000): Primary immunodeficiency diseases in Norway. J Clin Immunol 20: 477-485.

16. Cervato S, Mariniello B, Lazzarotto F, et al. (2009): Evaluation of the autoimmune regulator (AIRE) gene mutations in a cohort of Italian patients with autoimmune-polyendocrinopathy-candidiasis-ectodermal-dystrophy (APECED) and in their relatives. Clin Endocrinol (Oxf) 70: 421-428.

17. Walzer PD, Schultz MG, Western KA, Robbins JF (1976): Pneumocystis carinii pneumonia and primary immune deficiency diseases. Natl Cancer Inst Monogr 43: 65-74.

18. Ameratunga R, Lederman HM, Sullivan KE, et al. (1997): Defective antigen-induced lymphocyte proliferation in the X-linked hyper-IgM syndrome. J Pediatr 131: 147-150.

19. Mílledge J, Kakakios A, Gillis J, Fitzgerald DA (2003): Pneumocystis carinii pneumonia as a presenting feature of X-linked hyper-IgM syndrome. J Paediatr Child Health 39: 704-706.

20. Winkelstein JA, Marino MC, Johnston RB Jr, et al. (2000): Chronic granulomatous disease. Report on a national registry of 368 patients. Medicine (Baltimore) 79: 155-169.

21. Agarwal S, Cunningham-Rundles C (2009): Autoimmunity in common variable immunodeficiency. Curr Allergy Asthma Rep 9: 347-352.

22. Bussone G, Mouthon L (2009): Autoimmune manifestations in primary immune deficiencies. Autoimmun Rev 8: 332-336.

23. Gennery AR, Cant AJ (2009): New findings in primary immunodeficiency. Adv Exp Med Biol 634: 79-93.

24. Goyal R, Bulua AC, Nikolov NP, et al. (2009): Rheumatologic and autoimmune manifestations of primary immunodeficiency disorders. Curr Opin Rheumatol 21: 78-84.

25. Notarangelo LD, Gambineri E, Badolato R (2006): Immunodeficiencies with autoimmune consequences. Adv Immunol 89: 321-370.

26. Schuetz C, Niehues T, Friedrich W, Schwarz K (2010): Autoimmunity, autoinflammation and lymphoma in combined immunodeficiency (CID). Autoimmun Rev 9: 477-482.

27. Martínez JA, Piazuelo M, Almela M, et al. (2009): Evaluation of add-on devices for the prevention of phlebitis and other complications associated with the use of peripheral catheters in hospitalised adults: a randomised controlled study. J Hosp Infect 73: 135-142.

28. Glocker EO, Kotlarz D, Boztug K, et al. (2009): Inflammatory bowel disease and mutations affecting the interleukin-10 receptor. N Engl J Med 361: 2033-2045.

29. Subbarayan A, Colarusso G, Hughes SM, et al. (2011): Clinical features that identify children with primary immunodeficiency diseases. Pediatrics 127: 810-816. 